\title{
Imaging body composition in obesity and weight loss: challenges and opportunities
}

This article was published in the following Dove Press journal:

Diabetes, Metabolic Syndrome and Obesity:Targets and Therapy

25 September 2010

Number of times this article has been viewed

\author{
Heidi J Silver ${ }^{\prime}$ \\ E Brian Welch ${ }^{2}$ \\ Malcolm J Avison ${ }^{2}$ \\ Kevin D Niswender ${ }^{1,3}$ \\ 'Department of Medicine, \\ ${ }^{2}$ Department of Radiology and \\ Radiological Sciences, Institute \\ of Imaging Sciences, Vanderbilt \\ University, Nashville, TN, USA; \\ ${ }^{3}$ Tennessee Valley Healthcare System, \\ Nashville, TN, USA
}

\begin{abstract}
Obesity is a threat to public health worldwide primarily due to the comorbidities related to visceral adiposity, inflammation, and insulin resistance that increase risk for type 2 diabetes and cardiovascular disease. The translational research portfolio that originally described these risk factors was significantly enhanced by imaging techniques, such as dual-energy X-ray absorptiometry (DEXA), computed tomography (CT), and magnetic resonance imaging (MRI). In this article, we briefly review the important contributions of these techniques to understand the role of body composition in the pathogenesis of obesity-related complications. Notably, these imaging techniques have contributed greatly to recent findings identifying gender and racial differences in body composition and patterns of body composition change during weight loss. Although these techniques have the ability to generate good-quality body composition data, each possesses limitations. For example, DEXA is unable to differentiate type of fat, CT has better resolution but provides greater ionizing radiation exposure, and MRI tends to require longer imaging times and specialized equipment for acquisition and analysis. With the serious need for efficacious and cost-effective therapies to appropriately identify and treat at-risk obese individuals, there is greater need for translational tools that can further elucidate the interplay between body composition and the metabolic aberrations associated with obesity. In conclusion, we will offer our perspective on the evolution toward an ideal imaging method for body composition assessment in obesity and weight loss, and the challenges remaining to achieve this goal.
\end{abstract}

Keywords: obesity, imaging, body composition, visceral fat

\section{Introduction}

Obesity is an epidemic affecting at least 70 million people in the United States and over 300 million individuals worldwide. ${ }^{1}$ The public health consequences are enormous as obesity confers dramatically elevated risk for devastating comorbidities such as hypertension, diabetes, cardiovascular disease, liver disease, gallbladder disease, musculoskeletal disorders, and several types of cancer, in all population age groups. ${ }^{2}$ However, there is a developing appreciation that not all obese individuals and not all types of adiposity confer equivalent risk. ${ }^{3}$ Further, while the evidence indicates an intricate relationship among genotype, ethnicity, body composition, and cardiometabolic risk, current methods to characterize body composition have not kept pace with the evolving needs of clinicians and researchers. In this article, we briefly review the interplay between body composition, obesity, and cardiometabolic risk, with emphasis on the areas in which the evidence base is limited by the methods available, and recent advances that are likely to significantly impact the future of obesity investigation.
Correspondence: Heidi J Silver

Department of Medicine,

Vanderbilt University, Nashville, TN

37232-27I3, USA

Tel + I 6159361299

Email heidi.j.silver@vanderbilt.edu

and Kevin D Niswender

Department of Medicine,

Vanderbilt University, Nashville, TN

37232-27I3, USA

Email kevin.d.niswender@vanderbilt.edu 


\section{Body composition and metabolic risk}

Obesity-related cardiometabolic risk is often assessed by calculating body mass index (BMI), anthropometric measures such as waist and hip circumference, or other surrogate indicators of total adiposity. ${ }^{4}$ Although these techniques are useful in clinical practice to screen patients for disease risk and in large patient populations to determine associations with risk for comorbidities, especially when age and gender are considered, significant limitations exist. For example, data from the National Health and Nutrition Examination Survey III show that waist circumference cutoff values that correspond to the BMI range indicating overweight and obesity and risk for cardiovascular disease differ by ethnicity. ${ }^{5}$ That is, while the waist circumference values associated with overweight and obese BMI categories for Caucasian, African American, and Hispanic women appear similar, the corresponding waist circumference values are lower in African American and Hispanic men when compared to Caucasian men. In addition, these techniques are often not reliable for estimating an individual's percentage body fat. ${ }^{6}$ Moreover, they are unable to assess the amount, type (ie, subcutaneous, visceral, interstitial, or yellow marrow), and distribution of adipose tissue. Thus, the available clinical tools do not identify which components of body composition are the most important targets clinically or scientifically.

Consequently, imaging studies were critical in determining that the distribution of adipose tissue to central abdominal sites (ie, intra-abdominal or visceral adipose tissue [VAT]) is important in promoting risk for the inflammation, insulin resistance, and dyslipidemia associated with the development of comorbidities, ${ }^{7,8}$ as well as overall mortality. ${ }^{9,10}$ Nevertheless, a number of important questions remain. For example, there is scientific interest in a more comprehensive understanding of the metabolic, physiological, and pathophysiological differences among ethnic and life cycle groups, as well as a clearer understanding of the subset of overweight/obese individuals who have high BMIs and percentage body fat without the other features of the metabolic syndrome, ie, the "metabolically healthy but obese" phenotype. ${ }^{11-14}$ Fortunately, a number of additional body composition measurement techniques are being utilized in obesity research that promise to improve our understanding of existing biological concepts and generate new hypotheses.

\section{The need to advance body composition techniques}

The need for superior tools that provide direct measure of body composition becomes even more apparent when comparing differences in body composition and cardiometabolic risk by demographic factors like gender, age, and ethnicity. For example, in comparing males to females, it is well established that women have more total fat (adipose tissue), less total muscle (lean tissue), and a larger quantity of total adipose tissue in the lower trunk and pelvic region. ${ }^{15}$ However, when matched by BMI, men may have more visceral and hepatic adiposity. ${ }^{16,17}$ Clearly, these disparities in adipose tissue distribution confer different disease risks. There also exist differences in body composition and metabolic profile within genders - an effect that is most pronounced in women for whom significant differences occur across the life cycle. While women in their twenties and thirties are less likely to be obese, women at or beyond menopause have increased VAT. ${ }^{18}$ Moreover, among women of all age groups (adolescent, premenopausal, and postmenopausal), it has been shown that those who have a greater proportion of adiposity in the upper body have a greater likelihood of being dyslipidemic, glucose intolerant, hyperinsulinemic, and insulin resistant than those with greater proportion of adiposity in the lower body. ${ }^{19}$ While these risk profiles were determined by dualenergy X-ray absorptiometry (DEXA), the limitations in this technology include an inability to differentiate the type of adipose tissue, as will be discussed further.

Additionally, racial and ethnic differences in body composition have been identified in males and females. In fact, the relationship between abdominal adiposity and metabolic risk first came to light from studies using singleslice computed tomography (CT) scans of lean Japanese American males who, despite low BMI, had increased VAT and insulin resistance. ${ }^{20}$ Interestingly, in a study using wholebody multislice magnetic resonance imaging (MRI), no differences were detected in VAT between Asian American and Caucasian males after adjustment for age and total body fat. In contrast, Asian American females (over 30 years) had a higher proportion of VAT compared to Caucasian females. ${ }^{21}$ Prior studies also demonstrate puzzling differences in the characteristics of adiposity between African Americans and Caucasians. ${ }^{5,22-24}$ For example, African American women have less VAT and, therefore, more upper body subcutaneous adipose tissue (SAT) than Caucasian women, ${ }^{25,26}$ but they tend to be more insulin resistant. ${ }^{27}$ In fact, African Americans have worse cardiometabolic disease profiles overall: a higher incidence of hypertension, twice the prevalence of diabetes, and 2- to 3-fold greater incidences of coronary artery disease and stroke. Yet, greater upper body obesity is associated with mortality in Caucasians but not in African Americans. ${ }^{28}$ Hence, racial disparities in obesity and its comorbidities are 
not solely related to the anatomical location of fat (ie, upper vs lower body adiposity) or simply the relative proportion of VAT to SAT. This discrepancy has been recognized in the adjustments that have recently been made in waist circumference cutoffs to accommodate differences by race and ethnicity for cardiovascular risk. ${ }^{29}$

Overall, synthesis of published findings such as these suggest that while a number of potentially important findings have been uncovered, an underlying unifying mechanistic explanation has yet to emerge. We hypothesize that this gap in the evidence base may be related to the relative strengths and weaknesses of the body composition techniques that populate the literature. Hence, more sensitive methods to quantify metabolically active adipose depots promise to enhance mechanistic studies and our understanding of observed outcomes.

\section{Beyond adipose tissue: ectopic fat}

While absolute adipose mass, and perhaps the more insulin-resistant VAT, confers significant metabolic risk, the accumulation of fat in nonadipose tissues (ie, lipid within skeletal muscle and organs such as the liver, pancreas, and heart), for which in vivo quantification has been best achieved by advanced tools such as MR spectroscopy (MRS), is increasingly recognized as a mediator of insulin resistance and inflammation. ${ }^{30-34}$ In fact, in obesity, the association between intramyocellular lipid and insulin resistance is stronger than with VAT. ${ }^{33}$ It is also noteworthy that in states of reduced adipose tissue mass, eg, lipodystrophy, there is increased ectopic fat, insulin resistance, and diabetes. ${ }^{35}$

In conjunction with the focus on intramyocellular lipid, a novel fat depot existing between muscle bundles and beneath the muscle fascia, termed intermuscular adipose tissue (IMAT), may also reveal significant metabolic information. ${ }^{36}$ Interestingly, African Americans, who have more skeletal muscle than Caucasians, also appear to have a greater amount of IMAT. This disparity in ectopic fat appears most pronounced at higher levels of adiposity. ${ }^{36,37}$ Thus, it is conceivable that increased skeletal muscle and IMAT volume at least partially account for the observed differences among ethnicities in the insulin response to glucose. ${ }^{38}$ However, the extent to which IMAT functions in onset of insulin resistance remains to be further investigated. We suggest that a full understanding of the role of IMAT in health and disease will arise as a consequence of improved methods to quantify this lipid depot.

Similarly important, the accumulation of intrahepatic fat has serious implications due to its role in the pathogenesis of insulin resistance, dyslipidemia, steatosis, steatohepatitis, and progression to diabetes, coronary heart disease, and liver failure. ${ }^{39}$ While the current gold standard for diagnosis of "fatty liver" is liver biopsy, intensive efforts are underway to standardize both CT and MRI methods to quantify liver fat. Of course, MRS has also been used to understand liver lipid metabolism. Nevertheless, one of the limitations of current imaging methodologies, and an important opportunity to improve the scientific evidence base, would be the ability to incorporate measures of ectopic fat more widely in clinical and research settings.

\section{Body composition changes during weight loss}

Few studies have investigated how weight loss influences body composition. A classic model by Forbes ${ }^{40}$ predicts that during voluntary weight loss, the relative proportion of body mass loss would be approximately $80 \%$ fat and $20 \%$ lean tissue mass. The composition of body mass loss is likely influenced by many factors, including genetics, ${ }^{41}$ gender, ethnicity, aging, degree of energy deficit, ${ }^{42}$ dietary macronutrient composition and substrate utilization, ${ }^{43}$ hormonal status, ${ }^{44}$ degree and type of physical activity, ${ }^{45}$ comorbid disease states, and perhaps the type of weight loss intervention (dietary, pharmaceutical, or surgical). For example, it is expected that while males would lose proportionately more fat-free mass compared to females, they also lose more VAT. ${ }^{46}$ In response to caloric restriction, it has also been observed that Caucasian females lose approximately 50\% more VAT and less SAT than African Americans despite similar total weight loss. ${ }^{47}$ Intriguingly, these authors observed no significant differences between Caucasian and African American females with regard to improvements in insulin sensitivity and lipid profiles. ${ }^{48}$ While this finding appears to contrast with much other data, it suggests that the metabolic benefits of weight loss were not directly related to changes in the relative change in VAT and SAT, but it should be noted that the women studied were overweight, not obese, and premenopausal, and there was no assessment of the degree of ectopic fat and its role in the measured outcomes.

With involuntary weight loss, ie, that which occurs during chronic illness, injury, or malignancy, there is often an aberration in the proportional changes in body composition, yielding an excessive loss of lean tissue, and sometimes preservation of adipose tissue mass. For example, in studying overweight head and neck cancer patients treated with concomitant chemoradiation, we detected a body mass loss that was $72 \%$ lean and only $28 \%$ fat. ${ }^{49}$ Further, when 
negative energy balance is combined with reduced physical activity, such as that occurring with bed rest, space flight, or sometimes physiological aging, accelerated protein turnover results in greater lean tissue loss. ${ }^{50}$ From a metabolic perspective, this decrease in lean mass should have the detrimental consequence of impaired insulin sensitivity.

In reality, few populations have been prospectively studied with respect to the relative changes in fat vs lean tissue mass during voluntary or involuntary weight loss, not to mention changes in the type, distribution, and location of lipid burden. While it is expected that weight loss reduces fat and lean mass from various body depots, the mechanistic basis for such changes, whether adaptive or maladaptive, has not been clarified, and existing studies have utilized isotope-labeled tracer techniques that are not applicable outside highly sophisticated research settings. ${ }^{51}$ We suggest that the successful design of studies to better understand the interactions between body composition and cardiometabolic risk, and those capable of wholly assessing the efficacy of weight loss interventions, requires advanced techniques that measure not only the amount and type of adipose tissue but also its anatomical distribution. Moreover, ideal techniques must be capable of quantifying ectopic fat.

\section{Current imaging methods}

Imaging methods are considered to be the most accurate tools for measuring adipose tissue in body tissues and organs in clinical research. ${ }^{52,53}$ Imaging methods provide information about the spatial distribution of adipose tissue by exploiting differences in the imaging properties of different tissues and organs seen as 2-dimensional (2D) projections with DEXA scans or 2D multislice and 3-dimensional (3D) anatomical datasets with CT and MRI. Thus, regional differences in tissue X-ray absorption properties or MRI signal characteristics provide contrast in images that allow lean and adipose tissue to be distinguished and their respective volumes to be calculated. The accuracy of distinguishing tissue types depends on the degree of tissue contrast and the spatial resolution inherent to imaging modalities, which in turn depend primarily on the sensitivity and speed of acquiring images. Yet, no single method commonly employed accounts for all the factors (ie, amount, type, and distribution) that we and others hypothesize as highly relevant to outcomes of interest. Further, all methods contribute some form of error or bias that is usually related to assumptions about body composition that may not hold across different population groups and clinical scenarios. ${ }^{54}$ The pros and cons of the three common state-of-the-art imaging techniques for whole body measurement, ie, DEXA, CT, and MRI, ${ }^{55}$ will be contrasted in the following sections, with key characteristics summarized in Table 1.

However, before comparing body composition techniques, we must first recognize the important construct of categorizing body composition components into 5 distinct levels, ie, atomic level, molecular level, cellular level, tissue-systems level, and whole body level. While DEXA estimates body composition components at the molecular level (eg, fat mass), CT and MRI assess body composition components at the tissue-systems level (eg, adipose tissue). As this is not the focus of this article, we call the readers' attention to several excellent reviews further describing this fundamental concept. ${ }^{66-68}$

Table I Key characteristics of the most common imaging techniques used to quantify body composition

\begin{tabular}{|c|c|c|c|}
\hline Imaging technology & DEXA & Quantitative X-ray CT & MRI \\
\hline Year (first applied to body composition) & $|98|^{56}$ & $1979^{57}$ & $1984^{58}$ \\
\hline \multirow[t]{3}{*}{ Images acquired } & Coronal 2D projection & 2D single-slice & 2D single-slice \\
\hline & & & 2D multislice \\
\hline & & 2D multislice & 3D image volumes \\
\hline \multirow[t]{4}{*}{ Coefficient of variation } & Total body fat: $2 \%-3 \%^{59}$ & SAT: $0.50 \%^{60}$ & Total body fat: $2.90 \%{ }^{61}$ \\
\hline & & & VAT: $2.32 \%^{55}$ \\
\hline & & VAT: $1.20 \%{ }^{60}$ (multislice acquisition) & SAT: $2.25 \%{ }^{55}$ \\
\hline & & & TAT: $1.01 \% 55$ \\
\hline Scan time (whole body) & $10-20 \mathrm{~min}$ & Several seconds per slice & $10-20 \mathrm{~min}$ \\
\hline Resolution & $\mathrm{I} \mathrm{mm} \times 1 \mathrm{~mm}^{62}$ & $1.8 \mathrm{~mm} \times 1.8 \mathrm{~mm} \times 5 \mathrm{~mm}^{55}$ & $2 \mathrm{~mm} \times 2 \mathrm{~mm} \times 8 \mathrm{~mm}^{63}$ \\
\hline Radiation dose & $5-7 \mu \mathrm{Sv}^{59}$ & 2.7-10 mSv64,65 (whole body) & None \\
\hline Research cost per scan & Moderate $(\sim 135)$ & High $(\sim 500)$ & High $(\sim 500)$ \\
\hline Availability & Moderate & Limited & Limited \\
\hline
\end{tabular}

Abbreviations: DEXA, dual-energy X-ray absorptiometry; CT, computed tomography; MRI, magnetic resonance imaging; 2D, 2-dimensional; 3D, 3-dimensional; SAT, subcutaneous adipose tissue; VAT, visceral adipose tissue; TAT, total adipose tissue. 


\section{Dual-energy X-ray absorptiometry}

Replacing dual-photon absorptiometry (DPA) in 1989, DEXA was designed initially to estimate whole body and regional bone mineral density. DEXA uses an X-ray tube combined with a switch or filter to generate a larger flux of photons than DPA, yielding greater precision and shorter scan times. ${ }^{62}$ Typical DEXA scanners are composed of a flat table on which the subject lays supine with an X-ray generator under the table and a movable X-ray detector above that translates in the direction of head to foot. The X-ray source moving beneath the subject emits a pencil beam or fan beam of X-rays at two energy levels, typically $40 / 70$ or $43 / 110 \mathrm{keV}$ (depending on the manufacturer), which allow algorithms for the quantification of two distinct tissue types. While one energy level is well absorbed by soft tissue, ie, fat, the other is well absorbed by denser tissue, ie, bone. Hence, a technically well-acquired DEXA scan provides a reasonable estimate of total and regional (trunk, legs, and arms) fat and fat-free mass using proprietary equations based on tissue density and X-ray biophysics. Since total and regional fat estimated by DEXA is the sum of all fatty elements of soft tissue, a limitation of DEXA is the inability to differentiate adipose tissue and, thus, the inability to compare amounts or changes in VAT vs SAT.

Additionally, the trunk region determined by DEXA typically includes chest, abdomen, pelvis, and, thus, an inability to more specifically quantify the types of fat in specific body compartments. This can be particularly problematic in studying obese women as the determination of trunk fat by DEXA includes pelvic and gluteal fat, thereby confounding estimation of metabolic risk. ${ }^{69}$ Although some body composition researchers delineate the central abdominal region using DEXA by manually adjusting the trunk margins to include only the area from L2 to L4 vertebrae, this assessment still includes SAT. ${ }^{70}$ Further, defining the region of interest determined by DEXA is well-known to be operator dependent. Notably, with manipulation of the region of interest, it has been observed that DEXA estimates total abdominal adipose tissue better in nonobese (vs obese) persons. ${ }^{71}$

Nevertheless, percentage trunk fat estimated by DEXA has been positively correlated $(r=0.77)$ with VAT determined by CT in a sample of 206 lean and obese women. ${ }^{72}$ However, weaker correlations have been observed, supporting our hypothesis that while a number of techniques perform well across larger populations, there remains significant intraindividual variability. In another study, DEXA-estimated fat was correlated with MRI quantification of adipose tissue, but in $877 \mathrm{HIV}+$ and 260 healthy adults, DEXA significantly overestimated total and regional fat mass $(P<0.0001){ }^{73}$ Importantly, as the amount of body fat mass increased (ie, greater adiposity), the DEXA overestimates were proportionally larger, which would confound assessment of metabolic risk.

Mechanisms explaining some of these limitations of DEXA include variation based on the depth of tissue being analyzed. It appears that the anteroposterior thickness of a subject at $\leq 20 \mathrm{~cm}$ does not violate the assumption of density on which DEXA algorithms are based, and thus, at this thickness, overestimate of fat mass was $\leq 4 \%$ and overestimate of fat-free mass was $\leq 2 \%{ }^{74}$ However, at higher body thickness, DEXA provides greater error. ${ }^{73}$ This is an important consideration as most obese subjects will have anteroposterior thickness $>20 \mathrm{~cm}$.

Similarly, determination of lean soft tissue mass by DEXA (technically fat-free mass) suffers limitations. As lean mass in extremities is mostly muscle, DEXA-derived lean mass can predict skeletal muscle mass, although amounts are overestimated ${ }^{75}$ most likely because DEXA requires the potentially inaccurate assumption that there is a fixed proportion of muscle mass in the limbs. Hence, in a heterogeneous population of subjects with differing body composition, this assumption could confound the accuracy of skeletal muscle mass determination. Another fundamental assumption is that the soft tissue is normally hydrated (as DEXA does not estimate body water content). However, the presence of underhydration or overhydration is common in acute and chronic disease states, including obesity, and within certain life cycle groups, such as young children and older adults. It has been estimated that a 5\% change in the water content of the fat-free mass influences DEXA estimates of body fat about $2.5 \% .^{76}$ Even drinking water before scanning can increase estimates of total and lean mass. ${ }^{77}$

Therefore, depending on the manufacturer, hardware, software, and operator, different DEXA devices show differences in assessment of bone mineral content by $\pm 19 \%$, fat mass by $\pm 7 \%$, and lean mass by $\pm 4 \%{ }^{73,78,79}$ Nevertheless, the widespread availability and relative low expense of DEXA compared with more sophisticated imaging tools such as CT and MRI assure that DEXA will continue to be important for assessing body composition in health and disease, and therefore, it is scientifically important to understand its relative limitations.

More direct anatomical measures of both adipose and skeletal tissue include CT and MRI, both of which are able 
to resolve adipose from nonadipose tissue, although they are not typically used to detect the lipid burden within muscle unless additional spectroscopic methods are added to the procedure. A key advantage of these imaging techniques in body composition and obesity research is the ability to assess in vivo tissue type and distribution and the future potential for determining organ composition.

\section{CT scan}

CT systems are typically composed of a cylindrical housing unit that contains an X-ray tube source rotating in a plane perpendicular to the subject. Individuals enter the system on a moving table that travels through the gantry opening. The finite diameter of the gantry opening may limit the size of subjects that can be scanned. Like DEXA (although at much higher radiation doses), the X-ray source produces a pencil- or fan-shaped beam that passes through the body. The application of CT to body composition was first demonstrated in 1979 when the cross-sectional area of mid-arm muscle mass was quantified in persons ranging from $60 \%$ to $120 \%$ of ideal body weight. ${ }^{57}$ During the 1980 s, body composition research was further advanced when CT was used to quantify adipose tissue. ${ }^{80,81}$ These initial studies used a single-slice technique typically at the level of the umbilicus; now multislice techniques are most often utilized.

CT scans typically generate multiple 2D anatomical images that may form noncontiguous or contiguous 3D image volumes at a high resolution from which the difference in density between adipose vs nonadipose tissue can be quantified using tissue segmentation image analysis. From a CT image, tissue type can be distinguished, or segmented, based on CT number, which represents the electron density of the tissue within a particular volume element (voxel). CT numbers are expressed on a scale using Hounsfield units (HU), where $\mathrm{HU}=0$ for water and $\mathrm{HU}=-1,000$ for air. CT numbers for adipose tissue range from -190 to $-30 \mathrm{HU}$, whereas CT numbers for lean tissue range from 30 to $100 \mathrm{HU}$. Thus, CT provides both exquisite spatial and tissue-type resolution. ${ }^{64}$ Once segmented (manually or automatically), tissue area can be calculated using known in-plane dimensions of the CT image and tissue volume can be calculated using the slice thickness for a single slice, or a volume estimate can be made using the average cross-sectional area of two nearby slices and the distance between slices. For a contiguous set of slices, the volume calculation for each adjacent slice pair can be summed to yield a total volume. ${ }^{52}$ The total adipose tissue can then be estimated by using density values available in the literature, eg, $0.923 \mathrm{~kg} \mathrm{dm}{ }^{-3} \cdot{ }^{60}$ Recent studies have demonstrated excellent intraobserver and interobserver reproducibility of CT-based quantification of VAT and SAT.

While CT is excellent for segmenting adipose from nonadipose tissue, it is incapable of detecting ectopic fat. Further, there can be significant errors associated with the estimation of tissue and organ margins and reconstruction of volumetric data from noncontiguous 2D multislice datasets. Although a whole body protocol incorporating 28 slices positioned at specific locations has been reported, ${ }^{60}$ the time required for image post processing can be substantial for multislice acquisitions, taking up to 3 hours to analyze 28 slices. ${ }^{55}$

Most importantly, the amount of radiation exposure from CT limits its use in longitudinal data collection where serial measures would be necessary, and in vulnerable populations, such as growing children and women of child-bearing age. Typical protocols have limited the number of image slices to minimize radiation exposure. Indeed, the safety of radiation exposure from $\mathrm{CT}$ has received greater scrutiny from scientists recently because of the potential risk for free radical damage and cancer from radiation doses, which would vary in measuring whole body composition depending on the size of the subject and the type of CT equipment used. ${ }^{82}$ While no large epidemiological studies establishing the relative risk have been completed yet, recommendations have been made that MRI replace CT where possible.

\section{MR imaging}

The utility of MRI for assessing adipose tissue has been recognized for some time, but the low sensitivity and technical challenges including long scan duration have limited its broader utilization. Hence, early adopters used a small number of slices to assess adipose tissue. ${ }^{58,83}$ This evolved to the collection of multiple 2D slices from which whole body adipose and lean tissue volumes and masses were estimated using linear interpolation across consecutive slices. ${ }^{61,84,85}$ The movement from a few slices toward whole body imaging was further advanced with the recognition in 1998 that single-slice results from various body depots had significant variability in total and VAT across the range of BMI, which could not be predicted from determination of SAT or standard anthropometry. ${ }^{84}$

Although the contrast in CT images is generated by differences in the X-ray absorption properties of different tissues, the contrast in MRI scans depends on tissue-specific MR properties, such as proton density (ie, density of hydrogen atoms) and longitudinal (T1) and transverse 
$(\mathrm{T} 2, \mathrm{~T} 2 *)$ relaxation times. One or more of these properties can be exploited to generate contrast between lean and adipose tissues. For example, differences in the rate at which the magnetization of protons in fat and water return to equilibrium following an MR excitation (differences in fat and water T1s) leads to characteristic differences in intensities of the MR signal from fat (brighter) and water (darker) tissues in the T1-weighted MR images. This contrast in MRI scans can be exploited to provide greater discrimination of adipose and lean tissues and to provide improved estimates of ectopic lipid burden. Recently, measurement of adipose tissue using a whole-body T1-weighted imaging protocol was used to correlate VAT with insulin sensitivity in subjects at risk for type 2 diabetes. ${ }^{85}$ In this study, T1-weighted 3D whole body imaging was reported to have taken 20-25 minutes per subject. When shorter imaging times are preferred, fewer slices may be acquired to cover the entire body - as in a recently reported approach in which 10-mm-thick image slices were acquired every $40 \mathrm{~mm}$ of the whole body, but only $20 \%$ of the full body data was captured. ${ }^{73}$

Since MRI does not rely on ionizing radiation, it can be used in vulnerable population groups and in longitudinal studies requiring serial measures. And, MRI techniques have a slightly higher reproducibility than CT scans. For example, in healthy adults who had repeat scans during a 4-hour period, the coefficient of variation for VAT assessment by MRI was $10.6 \%$ compared with $12.8 \%$ with $\mathrm{CT}^{86}$ Improvements in MRI is evident in the more precise assessment of variability that has been determined using human cadavers and abdominal dissections, which showed tighter coefficient of variation ranging from $1 \%$ to $10 \%$ for SAT and from $6 \%$ to $11 \%$ for VAT measurements, ${ }^{87}$ and from $0.2 \%$ to $2.3 \%$ for appendicular skeletal muscle mass measurement. ${ }^{67}$

In addition to $\mathrm{T} 1$ differences, the different chemical environment of protons in water and in triglyceride fatty acid chains leads to small but measurable differences in their MRI resonance frequencies. The resonance frequency differences can be detected directly using MR spectroscopic techniques and indirectly with an imaging approach commonly known as the Dixon method. The Dixon method, introduced in $1984,{ }^{88}$ uses repeated acquisitions of the evolving MRI signal to capture images with echo times when water and fat signals are "in phase" (signals add) and "out of phase" (signals subtract). The MR signal echo time describes the time delay of signal acquisition after exciting the fat and water protons with radio frequency pulses. Pure water and fat images can theoretically be reconstructed from the sum and difference images to yield separated fat and water images, respectively.
The ratio of the fat to water signals at each point in the image is used to classify the tissue at that location as either adipose or lean. However, the separation of fat and water signals using the Dixon method can be confounded by imperfections in the scanner's static magnetic field (ie, B0 inhomogeneity), which causes ambiguities in the classification of MR signal as fat or water and error in the estimation of adipose and lean tissue volumes. Additionally, in areas with approximately equal fat and water signal intensities, the signal cancellation can impair estimation of the fat fraction. ${ }^{89}$

To address this problem, the Dixon method has been enhanced by increasing the number and modifying the spacing between the individual images acquired at different echo times..$^{90}$ Along with other refinements to the Dixon method over the past 2 decades,${ }^{91}$ such as using optimized asymmetric echo spacing ${ }^{89}$ and detailed modeling of differences in the patterns of MR signal decay of the fat and water components, more accurate estimates of adipose tissue and water within tissues have been produced. ${ }^{92}$

Because some protocols do not image the entire body, they are subject to various sources of error. First, when studying a body depot, such as the abdominal compartment, a practical challenge is how best to match slice positions across individuals with varying body habitus. Second, once a positioning algorithm has been established, there remains scan-to-scan variability in precision of slice placement. Third, even if slices were perfectly and reproducibly positioned, there are significant errors associated with the interpolation of volumes in unsampled interslice regions. This could be particularly problematic in longitudinal studies, and this artifact in interpolating volumes would be particularly acute in the visceral compartment where adipose tissue is heterogeneously distributed among other visceral organs. ${ }^{93}$

While using similar biophysical approaches as MRI related to chemical shift, MRS separates, characterizes, and records chemical signals within a single voxel to provide information about the actual composition of chemicals or metabolites within the image. The peaks of the metabolites are identified by their frequency and expressed as a shift in the frequency relative to a standard, typically water, which is highly concentrated in human tissues. This so-called chemical shift is expressed in parts per million (ppm). Thus, water is located at $4.26 \mathrm{ppm}$, whereas the dominant fat signal (from aliphatic protons) is located at approximately $1.25 \mathrm{ppm} .{ }^{95}$ Being independent of MR magnetic field strength, it allows comparisons of MR spectra obtained from imaging systems of different field strengths (eg, 1.5T vs 3T). The MR spectrum from a volume of tissue contains peaks at frequencies 
characteristic of the chemical (eg, water and fat) constituents. The relative areas of these peaks, after correction for the number of hydrogen atoms per molecule and differences in $\mathrm{T} 1, \mathrm{~T} 2$, etc, provide the relative concentrations of the different chemical species.

In human metabolic studies, MRS can yield greater chemical specificity than MRI, particularly in lean tissues (such as the liver), but this generally comes at the expense of spatial resolution and anatomical coverage. Furthermore, MRS is more sensitive to magnetic field inhomogeneities and motion (respiratory, visceral, bulk body) artifacts, making MRS especially challenging when assessing intra-abdominal tissues. For example, in the case of the pancreas, an irregularly shaped organ with a small volume, the larger voxel size required for MRS can compromise the tissue specificity of the MRS measurement. ${ }^{96}$ Like with MRI, correcting for artifacts such as respiratory motion using automated post processing is becoming more available. Ultimately, the information obtained by MRS is complimentary to that obtained by MRI, and as these methods continue to evolve, it will be ideal to be able to extract both types of information from a single MR dataset.

It is exciting that highly promising conceptual and technological advancements involving complete sampling of body compartment data, and thus, eliminating the need for interpolation, and minimizing the challenges associated with positioning have occurred over the past 2 years. In 2009 , a method for fully automated assessment of total adipose tissue was reported, which employed a continuously moving table, an optimized multi echo data acquisition sequence (an evolution of the Dixon method, termed "fat-water imaging"), and a computer algorithm for fully automated analysis of the data to yield total, visceral, and SAT measures. ${ }^{63}$ Such automated segmentation of adipose tissue compartments is critically important for widespread application of MR fat-water imaging. Strategies for segmentation include morphological approaches ${ }^{63}$ and those based on more advanced image processing such as fuzzy connectedness. ${ }^{94}$ Quantification of adipose tissue volume acquired on a voxelby-voxel basis with such techniques are more accurate and robust.

\section{Perspective on the promise of imaging to obesity, body composition, and metabolic disorders}

The application of novel methods to enhance imaging technology for improved assessment of human body composition promises to enhance our understanding of the mechanisms, physiology, and pathophysiology of obesityrelated comorbidities. This is especially critical in the current health care environment of increased acuity of care combined with substantial disparities in access to care and the need to contain health care resource use. As the presence of comorbidities varies among the obese, it is most important to be able to identify and target those obese individuals who are at risk for type 2 diabetes and cardiovascular disease. Yet, current methods of identifying these individuals have not achieved standardization, and thus, there is still no consensus on specific criteria to categorize obese phenotypes.

An ideal imaging method that incorporates the characteristics of being noninvasive, rapid, sensitive, and specific would have significant utility in addressing the limitations of current methodologies discussed earlier and enhance our understanding of metabolic risk in various populations. It is also critical that such a method be convenient and cost effective for use in both clinical and research settings. While calculating the costs of DEXA, CT, and MRI solely by the actual amount of time spent scanning makes these techniques comparable, the total costs remain significantly different because of the time and sophistication of methods needed to analyze the acquired data (Table 1). Nevertheless, a moving table fat-water MRI technique that enables acquisition of an entire noninterpolated whole body dataset, which is readily segmented using automated analysis techniques and could be performed on standard $1.5 \mathrm{~T}$ or $3 \mathrm{~T}$ body imagers in the range of 30 minutes or less, could meet this need, especially if the dataset acquired could also be used to extract the type of functional information provided from MRS of ectopic fat.

If the promise of such advances in imaging methodology can be met, coupling of these data with measures more typically utilized in clinical practice and research, such as blood pressure, lipid and liver enzyme profiles, biomarkers of inflammation (eg, high sensitivity c-reactive protein and PAI-1), and indicators of glucose metabolism and insulin sensitivity, would enable the development of more precise predictive risk models. Upon standardization, such a method could be utilized to distinguish differences among obese subjects across the span of gender, age, ethnicity, and BMI categories in both adipose and lean tissue amounts, types, and distribution in any body depot. Furthermore, and perhaps most critically, such methods might offer the potential to streamline therapeutic targets and offer critical insights into the efficacy of weight loss and other interventions designed to reduce metabolic risk, as well as increasing the utilization of body composition in obesity research. 


\section{Disclosure}

None of the authors have financial or scientific disclosures or conflicts with this publication.

\section{References}

1. Flegal KM, Carroll MD, Ogden CL, Curtin LR. Prevalence and trends in obesity among US adults, 1999-2008. JAMA. 2010;303: 235-241.

2. Eckel RH, Grundy SM, Zimmet PZ. The metabolic syndrome. Lancet. 2005;365:1415-1428.

3. Srdic B, Stokic E, Korac A, Ukropina M, Velickovic K, Breberina M. Morphological characteristics of abdominal adipose tissue in normalweight and obese women of different metabolic profiles. Exp Clin Endocrinol Diabetes. 2010; June 8 [Epub ahead of print]

4. Deurenberg P, Weststrate JA, Seidell JC. Body mass index as a measure of body fatness: age- and sex-specific prediction formulas. Br J Nutr. 1991;65:105-114.

5. Okosun IS, Tedders SH, Choi S, Dever GE. Abdominal adiposity values associated with established body mass indexes in white, black and hispanic Americans. A study from the Third National Health and Nutrition Examination Survey. Int J Obes Relat Metab Disord. 2000; 24:1279-1285.

6. Flegal KM, Shepherd JA, Looker AC, et al. Comparisons of percentage body fat, body mass index, waist circumference, and waist-stature ratio in adults. Am J Clin Nutr. 2009;89:500-508.

7. Despres JP, Lemieux I, Bergeron J, et al. Abdominal obesity and the metabolic syndrome: contribution to global cardiometabolic risk Arterioscler Thromb Vasc Biol. 2008;28:1039-1049.

8. Rexrode KM, Carey VJ, Hennekens CH, et al. Abdominal adiposity and coronary heart disease in women. JAMA. 1998;280:1843-1848.

9. Larsson B, Svardsudd K, Welin L, Wilhelmsen L, Bjorntorp P, Tibblin G. Abdominal adipose tissue distribution, obesity, and risk of cardiovascular disease and death: 13 year follow up of participants in the study of men born in 1913. Br Med J (Clin Res Ed). 1984;288: 1401-1404.

10. Filipovsky J, Ducimetiere P, Darne B, Richard JL. Abdominal body mass distribution and elevated blood pressure are associated with increased risk of death from cardiovascular diseases and cancer in middle-aged men. The results of a 15- to 20-year follow-up in the Paris prospective study I. Int J Obes Relat Metab Disord. 1993;17:197-203.

11. Sims EA. Are there persons who are obese, but metabolically healthy? Metabolism. 2001;50:1499-1504.

12. Brochu M, Tchernof A, Dionne IJ, et al. What are the physical characteristics associated with a normal metabolic profile despite a high level of obesity in postmenopausal women? J Clin Endocrinol Metab. 2001;86:1020-1025.

13. Messier V, Karelis AD, Robillard ME, et al. Metabolically healthy but obese individuals: relationship with hepatic enzymes. Metabolism. 2010;59:20-24.

14. Messier V, Karelis AD, Prud'homme D, Primeau V, Brochu M, Rabasa-Lhoret R. Identifying metabolically healthy but obese individuals in sedentary postmenopausal women. Obesity (Silver Spring). 2010;18:911-917.

15. Shen W, Punyanitya M, Silva AM, et al. Sexual dimorphism of adipose tissue distribution across the lifespan: a cross-sectional whole-body magnetic resonance imaging study. Nutr Metab (Lond) 2009;6:17.

16. Geer EB, Shen W. Gender differences in insulin resistance, body composition, and energy balance. Gend Med. 2009;6 Suppl 1:60-75.

17. Ogden CL, Carroll MD, Curtin LR, McDowell MA, Tabak CJ, Flegal KM. Prevalence of overweight and obesity in the United States, 1999-2004. JAMA. 2006;295:1549-1555.

18. Nedungadi TP, Clegg DJ. Sexual dimorphism in body fat distribution and risk for cardiovascular diseases. J Cardiovasc Transl Res. 2009; 2:321-327.
19. Williams MJ, Hunter GR, Kekes-Szabo T, Snyder S, Treuth MS Regional fat distribution in women and risk of cardiovascular disease. Am J Clin Nutr. 1997;65:855-860.

20. Fujimoto WY, Abbate SL, Kahn SE, Hokanson JE, Brunzell JD. The visceral adiposity syndrome in Japanese-American men. Obes Res. 1994;2:364-371

21. Park YW, Allison DB, Heymsfield SB, Gallagher D. Larger amounts of visceral adipose tissue in Asian Americans. Obes Res. 2001; 9:381-387.

22. Wagner DR, Heyward VH. Measures of body composition in blacks and whites: a comparative review. Am J Clin Nutr. 2000;71:1392-1402.

23. Dowling HJ, Fried SK, Pi-Sunyer FX. Insulin resistance in adipocytes of obese women: effects of body fat distribution and race. Metabolism. 1995;44:987-995.

24. Hyatt TC, Phadke RP, Hunter GR, Bush NC, Munoz AJ, Gower BA. Insulin sensitivity in African-American and white women: association with inflammation. Obesity (Silver Spring). 2009;17:276-282.

25. Conway JM, Yanovski SZ, Avila NA, Hubbard VS. Visceral adipose tissue differences in black and white women. Am J Clin Nutr. 1995;61: 765-771.

26. Albu JB, Murphy L, Frager DH, Johnson JA, Pi-Sunyer FX. Visceral fat and race-dependent health risks in obese nondiabetic premenopausal women. Diabetes. 1997;46:456-462.

27. Lovejoy JC, de la Bretonne JA, Klemperer M, Tulley R. Abdominal fat distribution and metabolic risk factors: effects of race. Metabolism. 1996;45:1119-1124.

28. Stevens J, Keil JE, Rust PF, Tyroler HA, Davis CE, Gazes PC. Body mass index and body girths as predictors of mortality in black and white women. Arch Intern Med. 1992;152:1257-1262.

29. Zhu S, Heymsfield SB, Toyoshima H, Wang Z, Pietrobelli A, Heshka S. Race-ethnicity-specific waist circumference cutoffs for identifying cardiovascular disease risk factors. Am J Clin Nutr. 2005;81: 409-415.

30. Manco M, Mingrone G, Greco AV, et al. Insulin resistance directly correlates with increased saturated fatty acids in skeletal muscle triglycerides. Metabolism. 2000;49:220-224.

31. Phillips DI, Caddy S, Ilic V, et al. Intramuscular triglyceride and muscle insulin sensitivity: evidence for a relationship in nondiabetic subjects. Metabolism. 1996;45:947-950.

32. Pan DA, Lillioja S, Kriketos AD, et al. Skeletal muscle triglyceride levels are inversely related to insulin action. Diabetes. 1997;46:983-988.

33. Goodpaster BH, Thaete FL, Kelley DE. Thigh adipose tissue distribution is associated with insulin resistance in obesity and in type 2 diabetes mellitus. Am J Clin Nutr. 2000;71:885-892.

34. Caro JF, Dohm LG, Pories WJ, Sinha MK. Cellular alterations in liver, skeletal muscle, and adipose tissue responsible for insulin resistance in obesity and type II diabetes. Diabetes Metab Rev. 1989;5: 665-689.

35. Heilbronn L, Smith SR, Ravussin E. Failure of fat cell proliferation, mitochondrial function and fat oxidation results in ectopic fat storage, insulin resistance and type II diabetes mellitus. Int J Obes Relat Metab Disord. 2004;28 Suppl 4:S12-S21.

36. Gallagher D, Kuznia P, Heshka S, et al. Adipose tissue in muscle: a novel depot similar in size to visceral adipose tissue. Am J Clin Nutr. 2005;81:903-910.

37. Yim JE, Heshka S, Albu J, et al. Intermuscular adipose tissue rivals visceral adipose tissue in independent associations with cardiovascular risk. Int J Obes (Lond). 2007;31:1400-1405.

38. Albu JB, Kovera AJ, Allen L, et al. Independent association of insulin resistance with larger amounts of intermuscular adipose tissue and a greater acute insulin response to glucose in African American than in white nondiabetic women. Am J Clin Nutr. 2005;82: $1210-1217$

39. Koska J, Stefan N, Permana PA, et al. Increased fat accumulation in liver may link insulin resistance with subcutaneous abdominal adipocyte enlargement, visceral adiposity, and hypoadiponectinemia in obese individuals. Am J Clin Nutr. 2008;87:295-302. 
40. Forbes GB. Lean body mass-body fat interrelationships in humans. Nutr Rev. 1987;45:225-231.

41. Forbes GB, Sauer EP, Weitkamp LR. Lean body mass in twins. Metabolism. 1995;44:1442-1446.

42. Forbes GB. Body fat content influences the body composition response to nutrition and exercise. Ann N Y Acad Sci. 2000;904:359-365.

43. Hall KD, Bain HL, Chow CC. How adaptations of substrate utilization regulate body composition. Int J Obes (Lond). 2007;31:1378-1383.

44. Volek JS, Sharman MJ, Love DM, et al. Body composition and hormonal responses to a carbohydrate-restricted diet. Metabolism. 2002;51:864-870.

45. Janssen I, Fortier A, Hudson R, Ross R. Effects of an energy-restrictive diet with or without exercise on abdominal fat, intermuscular fat, and metabolic risk factors in obese women. Diabetes Care. 2002;25: 431-438.

46. Redman LM, Heilbronn LK, Martin CK, Alfonso A, Smith SR, Ravussin E. Effect of calorie restriction with or without exercise on body composition and fat distribution. J Clin Endocrinol Metab. 2007;92: 865-872.

47. Weinsier RL, Hunter GR, Gower BA, Schutz Y, Darnell BE, Zuckerman PA. Body fat distribution in white and black women: different patterns of intraabdominal and subcutaneous abdominal adipose tissue utilization with weight loss. Am J Clin Nutr. 2001;74:631-636.

48. Gower BA, Weinsier RL, Jordan JM, Hunter GR, Desmond R. Effects of weight loss on changes in insulin sensitivity and lipid concentrations in premenopausal African American and white women. Am J Clin Nutr. 2002;76:923-927.

49. Silver HJ, Dietrich MS, Murphy BA. Changes in body mass, energy balance, physical function, and inflammatory state in patients with locally advanced head and neck cancer treated with concurrent chemoradiation after low-dose induction chemotherapy. Head Neck. 2007;29:893-900.

50. Biolo G, Ciocchi B, Stulle M, et al. Calorie restriction accelerates the catabolism of lean body mass during 2 wk of bed rest. Am J Clin Nutr. 2007;86:366-372.

51. Mittendorfer B, Patterson BW, Klein S. Effect of weight loss on VLDLtriglyceride and apoB-100 kinetics in women with abdominal obesity. Am J Physiol Endocrinol Metab. 2003;284:E549-E556.

52. Bray G, Bouchard C, James WPT. Handbook of Obesity. New York: Marcel Dekker; 1998.

53. Ross R. Advances in the application of imaging methods in applied and clinical physiology. Acta diabetol. 2003;40 Suppl 1:S45-S50.

54. Lee SY, Gallagher D. Assessment methods in human body composition. Curr Opin Clin Nutr Metab Care. 2008;11:566-572.

55. Kullberg J, Brandberg J, Angelhed JE, et al. Whole-body adipose tissue analysis: comparison of MRI, CT and dual energy X-ray absorptiometry. Br J Radiol. 2009;82:123-130.

56. Peppler WW, Mazess RB. Total body bone mineral and lean body mass by dual-photon absorptiometry. I. Theory and measurement procedure. Calcif Tissue Int. 1981;33:353-359.

57. Heymsfield SB, Olafson RP, Kutner MH, Nixon DW. A radiographic method of quantifying protein-calorie undernutrition. Am J Clin Nutr. 1979;32:693-702.

58. Foster MA, Hutchison JM, Mallard JR, Fuller M. Nuclear magnetic resonance pulse sequence and discrimination of high- and low-fat tissues. Magn Reson Imaging. 1984;2:187-192.

59. Andreoli A, Scalzo G, Masala S, Tarantino U, Guglielmi G. Body composition assessment by dual-energy X-ray absorptiometry (DXA). Radiol Med. 2009;114:286-300.

60. Chowdhury B, Sjöström L, Alpsten M, Kostanty J, Kvist H, Löfgren R. A multicompartment body composition technique based on computerized tomography. Int J Obes Relat Metab Disord. 1994;18: 219-234.

61. Ross R, Léger L, Morris D, de Guise J, Guardo R. Quantification of adipose tissue by MRI: relationship with anthropometric variables. J Appl Physiol. 1992;72:787-795.
62. Sartoris DJ, Resnick D. Dual-energy radiographic absorptiometry for bone densitometry: current status and perspective. AJR Am J Roentgenol. 1989;152:241-246.

63. Kullberg J, Johansson L, Ahlström H, et al. Automated assessment of whole-body adipose tissue depots from continuously moving bed MRI: a feasibility study. J Magn Reson Imaging. 2009;30:185-193.

64. Mattsson Sr, Thomas B. Development of methods for body composition studies. Phys Med Biol. 2006;51:R203-R228.

65. Maurovich-Horvat P, Massaro J, Fox CS, Moselewski F, O'Donnell CJ, Hoffmann U. Comparison of anthropometric, area- and volume-based assessment of abdominal subcutaneous and visceral adipose tissue volumes using multi-detector computed tomography. Int J Obes (Lond). 2007;31:500-506.

66. Wang ZM, Pierson RN Jr, Heymsfield SB. The five-level model: a new approach to organizing body-composition research. Am J Clin Nutr. 1992;56:19-28.

67. Heymsfield SB, Wang Z, Baumgartner RN, Ross R. Human body composition: advances in models and methods. Annu Rev Nutr. 1997; 17:527-558.

68. Shen W, Wang Z, Punyanita M, et al. Adipose tissue quantification by imaging methods: a proposed classification. Obes Res. 2003;11:5-16.

69. Sierra-Johnson J, Johnson BD, Bailey KR, Turner ST. Relationships between insulin sensitivity and measures of body fat in asymptomatic men and women. Obes Res. 2004;12:2070-2077.

70. Carey DG, Jenkins AB, Campbell LV, Freund J, Chisholm DJ. Abdominal fat and insulin resistance in normal and overweight women: direct measurements reveal a strong relationship in subjects at both low and high risk of NIDDM. Diabetes. 1996;45:633-638.

71. Kamel EG, McNeill G, Han TS, et al. Measurement of abdominal fat by magnetic resonance imaging, dual-energy $\mathrm{X}$-ray absorptiometry and anthropometry in non-obese men and women. Int J Obes Relat Metab Disord. 1999;23:686-692.

72. Treuth MS, Hunter GR, Kekes-Szabo T. Estimating intraabdominal adipose tissue in women by dual-energy X-ray absorptiometry. Am J Clin Nutr. 1995;62:527-532.

73. Scherzer R, Shen W, Bacchetti P, et al. Comparison of dual-energy $\mathrm{X}$-ray absorptiometry and magnetic resonance imaging-measured adipose tissue depots in HIV-infected and control subjects. Am J Clin Nutr. 2008;88:1088-1096.

74. Fuller NJ, Laskey MA, Elia M. Assessment of the composition of major body regions by dual-energy X-ray absorptiometry (DEXA), with special reference to limb muscle mass. Clin Physiol. 1992;12: 253-266.

75. Wang ZM, Visser M, Ma R, et al. Skeletal muscle mass: evaluation of neutron activation and dual-energy X-ray absorptiometry methods. J Appl Physiol. 1996;80:824-831.

76. Salamone LM, Fuerst T, Visser M, et al. Measurement of fat mass using DEXA: a validation study in elderly adults. J Appl Physiol. 2000;89:345-352.

77. Thomsen TK, Jensen VJ, Henriksen MG. In vivo measurement of human body composition by dual-energy X-ray absorptiometry (DXA). Eur $J$ Surg. 1998;164:133-137.

78. Kullberg J, Brandberg J, Angelhed JE, et al. Whole-body adipose tissue analysis: comparison of MRI, CT and dual energy X-ray absorptiometry. Br J Radiol. 2009;82:123-130.

79. Genton L, Hans D, Kyle UG, Pichard C. Dual-energy X-ray absorptiometry and body composition: differences between devices and comparison with reference methods. Nutrition. 2002;18:66-70.

80. Borkan GA, Gerzof SG, Robbins AH, Hults DE, Silbert CK, Silbert JE. Assessment of abdominal fat content by computed tomography. Am J Clin Nutr. 1982;36:172-177.

81. Sjostrom L, Kvist H, Cederblad A, Tylen U. Determination of total adipose tissue and body fat in women by computed tomography, $40 \mathrm{~K}$, and tritium. Am J Physiol. 1986;250:E736-E745.

82. Brenner DJ, Hall EJ. Computed tomography - an increasing source of radiation exposure. $N$ Engl J Med. 2007;357:2277-2284. 
83. Hayes PA, Sowood PJ, Belyavin A, Cohen JB, Smith FW. Sub-cutaneous fat thickness measured by magnetic resonance imaging, ultrasound, and calipers. Med Sci Sports Exerc. 1988;20:303-309.

84. Thomas EL, Saeed N, Hajnal JV, et al. Magnetic resonance imaging of total body fat. J Appl Physiol. 1998;85:1778-1785.

85. Machann J, Thamer C, Schnoedt B, et al. Standardized assessment of whole body adipose tissue topography by MRI. J Magn Reson Imaging. 2005;21:455-462.

86. Seidell JC, Bakker CJ, van der Kooy K. Imaging techniques for measuring adipose-tissue distribution - a comparison between computed tomography and 1.5-T magnetic resonance. Am J Clin Nutr. 1990; 51:953-957.

87. Abate N, Burns D, Peshock RM, Garg A, Grundy SM. Estimation of adipose tissue mass by magnetic resonance imaging: validation against dissection in human cadavers. J Lipid Res. 1994;35:1490-1496.

88. Dixon WT. Simple proton spectroscopic imaging. Radiology. 1984;153:189-194.

89. Reeder S, Pineda A, Wen Z, et al. Iterative decomposition of water and fat with echo asymmetry and least-squares estimation (IDEAL) application with fast spin-echo imaging. Magn Reson Med. 2005;54: 636-644.
90. Glover GH, Schneider E. Three-point Dixon technique for true water/fat decomposition with B0 inhomogeneity correction. Magn Reson Med. 1991;18:371-383.

91. Ma J. Dixon techniques for water and fat imaging. J Magn Reson Imaging. 2008;28:543-558.

92. Brodsky EK, Holmes JH, Yu H, Reeder SB. Generalized k-space decomposition with chemical shift correction for non-Cartesian waterfat imaging. Magn Reson Med. 2008;59:1151-1164.

93. Kullberg J, Brandberg J, Angelhed JE, et al. Whole-body adipose tissue analysis: comparison of MRI, CT and dual energy X-ray absorptiometry. Br J Radiol. 2009;82:123-130.

94. Jin Y, Imielinska C, Laine AF, Udupa J, Shen W, Heymsfield S. Segmentation and evaluation of adipose tissue from whole body MRI scans. In: Larsen R, Nielsen M, Sporring J, editors. Medical Image Computing and Computer-Assisted Intervention. Berlin: Springer; 2003:635-642.

95. Qayyum A. MR spectroscopy of the liver: principles and clinical applications. Radiographics. 2009;29:1653-1664.

96. Hu H, Kim H-W, Nayak K, Goran M. Comparison of fat-water MRI and single-voxel MRS in the assessment of hepatic and pancreatic fat fractions in humans. Obesity (Silver Spring). 2010;18:841-847.

\section{Publish your work in this journal}

Diabetes, Metabolic Syndrome and Obesity: Targets and Therapy is an international, peer-reviewed open-access journal committed to the rapid publication of the latest laboratory and clinical findings in the fields of diabetes, metabolic syndrome and obesity research. Original research, review, case reports, hypothesis formation, expert opinion and commentaries are all considered for publication. The manuscript management system is completely online and includes a very quick and fair peer-review system, which is all easy to use. Visit http://www.dovepress.com/testimonials.php to read real quotes from published authors.

Submit your manuscript here: http://www.dovepress.com/diabetes-metabolic-syndrome-and-obesity-targets-and-therapy-journal 